TRANSACTIONS OF THE

AMERICAN MATHEMATICAL SOCIETY

Volume 353, Number 1, Pages 1-20

S 0002-9947(00)02632-5

Article electronically published on August 8, 2000

\title{
STANDARD REALIZATIONS OF CRYSTAL LATTICES VIA HARMONIC MAPS
}

\author{
MOTOKO KOTANI AND TOSHIKAZU SUNADA
}

\begin{abstract}
An Eells-Sampson type theorem for harmonic maps from a finite weighted graph is employed to characterize the equilibrium configurations of crystals. It is thus observed that the mimimum principle frames symmetry of crystals.
\end{abstract}

\section{INTRODUCTION}

We shall start with the motivation behind the subject which we are concerned within this paper. A simplified model of crystals is a system of springs which connect masses distributed periodically in Euclidean space. The masses represent atoms, and the springs represent symbolically the internal forces binding two atoms in the crystal. A periodic structure of the system, which shows a peculiar feature of crystals, is described as invariance of the system under the action of an additive group of translations in the space. The smallest pattern unit of the crystal from which the whole crystal can be built up by translations is called a primitive unit cell of the system. The atoms in a real crystal are vibrating around their equilibrium positions with thermal motion. The atoms are supposed to rest at the equilibrium positions when the absolute temperature is zero.

The main problem considered in this paper is to find a geometric characterization for the equilibrium configurations of the atoms in the crystal. For instance, we ask why the crystal of diamond has such a beautiful configuration of the carbon atoms which assures the rigid structure of diamond (Figure 1 in \$3).

This rather fundamental and simple question seems to have not yet been answered in full generality. The classical theory of crystallographic groups, a treatment of crystals from the viewpoint of symmetries and having a long history since 1830 , does not give any satisfactory answer to the question. We have a macroscopic characterization of equilibrium shapes of crystals due to G.Wulff (1901), which also fails to give any microscopic characterization for configurations of the atoms in crystals.

An equilibrium configuration should satisfy the condition that the internal forces in the crystal are in equilibrium; that is, the total force acting on any atom from its nearest neighbors vanishes. This condition, however, is not enough to determine the equilibrium configuration uniquely. Actually, as is easily seen, the equilibrium of the internal forces is preserved by any affine transformation of the space.

Received by the editors March 8, 1999.

2000 Mathematics Subject Classification. Primary 58E20, 58E11, 58E30; Secondary 82B99.

Key words and phrases. Crystal lattice, harmonic map, Albanese map, Albanese torus, abelian covering, weighted graph. 
As a characterization of equilibrium configurations, we shall take up the "minimum principle" for the potential energy per primitive unit cell with a fixed volume, which seems natural from physical and mathematical points of view. Indeed, we establish the following theorem.

Theorem 1. Among configurations of a given crystal having a primitive unit cell with a fixed volume, there exists a configuration with the minimal potential energy per primitive unit cell, and it is uniquely determined up to motions of the space.

The proof of the above theorem is effectively reduced to the theory of harmonic maps from finite weighted graphs into flat tori, as will be explained in \$3. In fact, we shall prove the following theorem which is regarded as a geometric version of the statement in Theorem 1

Theorem 2. Let $X_{0}$ be a finite connected weighted graph, $\mathcal{T}$ a torus, and $C$ a homotopy class of piecewise smooth maps from $X_{0}$ into $\mathcal{T}$ such that a map in $C$ induces the surjective homomorphism of $\pi_{1}\left(X_{0}\right)$ onto $\pi_{1}(\mathcal{T})$. For a given positive constant $v_{0}$, there exists a pair $\left(\Phi_{0}, g_{0}\right)$, consisting of a harmonic map $\Phi_{0} \in C$ and a flat metric $g_{0}$ on $\mathcal{T}$ such that $\operatorname{vol}\left(\mathcal{T}, g_{0}\right)=v_{0}$, satisfying

$$
E\left(\Phi_{0}, g_{0}\right) \leq E(\Phi, g)
$$

for every $\Phi \in C$ and every flat metric $g$ on $\mathcal{T}$ with $\operatorname{vol}(\mathcal{T}, g)=v_{0}$, where $E(\Phi, g)$ denotes the energy of the map $\Phi: X_{0} \rightarrow(\mathcal{T}, g)$. The pair $\left(\Phi_{0}, g_{0}\right)$ is determined uniquely. More precisely, if $E(\Phi, g)=E\left(\Phi_{0}, g_{0}\right)$, then $g=g_{0}$ and $\Phi$ coincides with a translation of $\Phi_{0}$.

The terminology used in the above theorem will be introduced in $\$ 2$ In $\$ 4$ we characterize the pair $\left(\Phi_{0}, g_{0}\right)$ in terms of a graph-theoretical analogue of the Albanese maps into Albanese tori, a concept originally introduced in algebraic geometry, and give a standard way to realize the configuration of the crystal satisfying the minimum principle.

As will be seen in 5 , the classical configurations for many crystals in two and three dimension turn out to satisfy the minimum principle. The diamond crystal illustrates this characterization.

It should be pointed out that one may establish an analogue of Theorem 2 for harmonic maps from a compact Riemannian manifold into a flat torus.

Let us conclude this introduction by mentioning an unexpected relation of the standard realization of a crystal lattice $X$ to the asymptotic behavior of the transition probability of a random walk on $X$ (see [8] for the details).

Theorem. Consider a reversible random walk on a crystal lattice $X$ with an invariant measure $m$. We equip $X$ with a canonical weighted structure. Let $d(x, y)$ be the distance on $X$ induced from the Euclidean distance by the standard realization of $X$ in $\mathbb{R}^{k}$. If $X$ is not bipartite, then there exist positive constants $C_{1}, C_{2}$ such that, for the n-step transition probability $p(n, x, y)$,

$$
\lim _{n \uparrow \infty}\left[(4 \pi n)^{k / 2} p(n, x, y) m(y)^{-1}-C_{1} \exp \left(-\frac{C_{2}}{n} d(x, y)^{2}\right)\right]=0
$$

uniformly for all $x, y \in X$.

Acknowledgement. The authors would like to thank H. Urakawa for informing them of his and other recent works on harmonic maps between singular spaces. 


\section{HARMONIC MAPS OF A WEIGHTED GRAPH INTO A RIEMANIAN MANIFOLD}

The notion of harmonic maps was introduced by J. Eells and J.H. Sampson in 1964 in their fundamantal paper [2] as a generalization of geodesics, harmonic functions and holomorphic mappings between Kähler manifolds. As in the case of ordinary harmonic maps of Riemannian manifolds, harmonic maps of a finite weighted graph $X$ into a Riemannian manifold $Y$ are defined to be critical maps for the energy functional $E$, where the graph $X$ is regarded as a 1-dimensional singular space, or precisely speaking, a 1-dimensional CW-complex, and maps are always supposed to be piecewise smooth. We shall explain these notions in detail.

Let $X=(V, E)$ be a connected locally finite graph with a set $V$ of vertices and a set $E$ of all oriented edges. For an edge $e \in E$, we denote by $o(e)$ and $t(e)$ the origin and the terminus of $e$ repectively. The inverse edge of $e$ will be denoted by $\bar{e}$. Consider positive valued functions $m_{V}$ on $V$ and $m_{E}$ on $E$, where it is assumed that $m_{E}(\bar{e})=m_{E}(e)$ for every $e \in E$. The functions $m_{V}$ and $m_{E}$ are said to be weight functions on $V$ and $E$ respectively. A graph with weight functions is called a weighted graph.

We first fix a way to identify the graph $X$ with a CW-complex. Take the disjoint union $V \amalg(E \times[0,1])$ and introduce the equivalence relation $\sim$ defined by

$$
\begin{aligned}
o(e) & \sim(e, 0) \\
t(e) & \sim(e, 1), \\
(e, t) & \sim(\bar{e}, 1-t) \text { for } 0 \leq t \leq 1
\end{aligned}
$$

Then the CW-complex associated with the graph $X$ is defined as the quotient space $V \amalg(E \times[0,1]) / \sim$ so that the unoriented edges (resp. vertices) are identified with 1cells (resp. 0-cells). The CW-complex $X$ is considered as a one-dimensional singular Riemanninan manifold with the metric $d s^{2}=m_{E}^{2}(e) d t^{2}$ on each edge $e \in E$.

Given a map $\Phi$ of $X$ into a Riemannian manifold $(Y, g)$, we put $\Phi_{e}(t)=\Phi(e, t)$ for $e \in E$ and $0 \leq t \leq 1$; this is considered as the restriction of $\Phi$ to the edge $e$. The map $\Phi$ is said to be piecewise smooth if $\Phi$ is continuous and the curve $\Phi_{e}$ is smooth in $t$ for every $e \in E$.

For a piecewise smooth map $\Phi$ of a finite weighted graph $X$ into $(Y, g)$, define the energy of $\Phi$ by

$$
E(\Phi)=E(\Phi, g)=\frac{1}{2} \sum_{e \in E} m_{E}(e) \int_{0}^{1}\left\|\frac{d \Phi_{e}}{d t}\right\|^{2} d t
$$

We shall compute the first and the second variation formulae. Let $\Phi(u, \cdot)(|u|<\varepsilon)$ be a smooth variation of of a map $\Phi=\Phi(0, \cdot)$, and put

$$
\frac{d \Phi_{e}}{d t}=\dot{\Phi}_{e}, \quad \frac{\partial \Phi_{e}}{\partial u}(u, t)=W_{e}(t), \quad \frac{\partial \Phi_{e}}{\partial u}(u, 0)=W(x)
$$

where we abuse the notation and write $\Phi_{e}=\Phi_{e}(u, t)$. 
Theorem 2.1 (The first and second variation formulae). At $u=0$, we have

$$
\begin{aligned}
\frac{d E(\Phi)}{d u}= & -2 \sum_{x \in V}\left\langle W(x), \sum_{e \in E_{x}} m_{E}(e) \dot{\Phi}_{e}(0)\right\rangle \\
& -\sum_{e \in E} m_{E}(e) \int_{0}^{1}\left\langle W_{e}(t), \frac{D}{d t} \dot{\Phi}_{e}\right\rangle d t \\
\frac{d^{2} E(\Phi)}{d u^{2}}= & -2 \sum_{x \in V}\left\langle D_{W} W(x), \sum_{e \in E_{x}} m_{E}(e) \dot{\Phi}_{e}(0)\right\rangle \\
& +\sum_{e \in E} m_{E}(e) \int_{0}^{1}\left(-\left\langle D_{W_{e}} W_{e}, \frac{D}{d t} \dot{\Phi}_{e}\right\rangle\right. \\
& \left.+\left\|\frac{D W_{e}}{d t}\right\|^{2}-\left\langle W_{e}, R\left(W_{e}, \dot{\Phi}_{e}\right) \dot{\Phi}_{e}\right\rangle\right) d t
\end{aligned}
$$

where $E_{x}=\{e \in E \mid o(e)=x\}$ and $R$ denotes the curvature tensor.

Proof. By routine computation, we have

$$
\frac{d E(\Phi)}{d u}=\sum_{e \in E} m_{E}(e)\left(\left.\left\langle\frac{\partial \Phi_{e}}{\partial u}, \frac{\partial \Phi_{e}}{\partial t}\right\rangle\right|_{t=0} ^{t=1}-\int_{0}^{1}\left\langle\frac{\partial \Phi_{e}}{\partial u}, \frac{D}{\partial t} \frac{\partial \Phi_{e}}{\partial t}\right\rangle d t\right) .
$$

Since

$$
\begin{aligned}
& \frac{\partial \Phi_{\bar{e}}}{\partial u}(0,1)=\frac{\partial \Phi_{e}}{\partial u}(0,0)=W(o(e)), \quad \frac{\partial \Phi_{\bar{e}}}{\partial t}(0,1)=-\frac{\partial \Phi_{e}}{\partial t}(0,0)=-\dot{\Phi}_{e}(0), \\
& \text { (9) } \sum_{e \in E} m_{E}(e)\left\langle\frac{\partial \Phi_{e}}{\partial u}, \frac{\partial \Phi_{e}}{\partial t}\right\rangle(0,1) \\
& =\sum_{e \in E} m_{E}(\bar{e})\left\langle\frac{\partial \Phi_{\bar{e}}}{\partial u}, \frac{\partial \Phi_{\bar{e}}}{\partial t}\right\rangle(0,1)=-\sum_{e \in E} m_{E}(e)\left\langle\frac{\partial \Phi_{e}}{\partial u}, \frac{\partial \Phi_{e}}{\partial t}\right\rangle(0,0),
\end{aligned}
$$

we find that

$$
\frac{d E(\Phi)}{d u}(0)=-\sum_{e \in E} m_{E}(e)\left(2\left\langle W(o(e)), \dot{\Phi}_{e}(0)\right\rangle+\int_{0}^{1}\left\langle W_{e}, \frac{D}{d t} \dot{\Phi}_{e}\right\rangle d t\right),
$$

from which the first variation formula easily follows.

By a similar argument, we obtain the second variation formula

$$
\begin{aligned}
\frac{d^{2} E(\Phi)}{d u^{2}}(0)= & \sum_{e \in E} m_{E}(e)\left(\left.\left\langle D_{W_{e}} W_{e}, \dot{\Phi}_{e}\right\rangle\right|_{t=0} ^{t=1}+\int_{0}^{1}\left(-\left\langle D_{W_{e}} W_{e}, \frac{D}{d t} \dot{\Phi}_{e}\right\rangle\right.\right. \\
& \left.\left.+\left\|\frac{D W_{e}}{d t}\right\|^{2}-\left\langle W_{e}, R\left(W_{e}, \dot{\Phi}_{e}\right) \dot{\Phi}_{e}\right\rangle\right) d t\right) \\
= & -2 \sum_{x \in V}\left\langle D_{W} W(x), \sum_{e \in E_{x}} m_{E}(e) \dot{\Phi}_{e}\right\rangle \\
& +\sum_{e \in E} m_{E}(e) \int_{0}^{1}\left(-\left\langle D_{W_{e}} W_{e}, \frac{D}{d t} \dot{\Phi}_{e}\right\rangle\right. \\
& \left.+\left\|\frac{D W_{e}}{d t}\right\|^{2}-\left\langle W_{e}, R\left(W_{e}, \dot{\Phi}_{e}\right) \dot{\Phi}_{e}\right\rangle\right) d t
\end{aligned}
$$


Applying the first variation formula, we obtain

Theorem 2.2. A piecewise smooth map $\Phi$ is a critical map if and only if $\Phi$ is a piecewise geodesic map, i.e. $\Phi_{e}$ is a geodesic for every edge $e$, and at each $x \in V$

$$
\sum_{e \in E_{x}} m_{E}(e) \dot{\Phi}_{e}(0)=0
$$

A piecewise geodesic map of a general weighted graph $X$ into $Y$ satisfying (12) is called harmonic.

Remark. The energy of a piecewise geodesic map $\Phi$ is equal to

$$
E(\Phi)=\frac{1}{2} \sum_{e \in E} m_{E}(e) \ell(e)^{2},
$$

where $\ell(e)$ is the length of the geodesic $\Phi_{e}$.

In the special case when the target manifold $Y$ is the Euclidean space $\mathbb{R}^{n}$, a piecewise geodesic map $\Phi$ of $X$ into $Y$ is uniquely determined by its restriction to the set of vertices, and the condition (12) is rewritten as

$$
\sum_{e \in E_{x}} m_{E}(e)(\Phi(t(e))-\Phi(o(e)))=0,
$$

since $\dot{\Phi}_{e}(0)=\Phi(t(e))-\Phi(o(e))$.

Define the discrete Laplacian $\Delta$ acting on vector-valued functions on the set of vertices by

$$
\Delta f(x)=m_{V}(x)^{-1} \sum_{e \in E_{x}} m_{E}(e)(f(t(e))-f(o(e))) .
$$

With this terminology, (14) is rephrased as

$$
\Delta \Phi=0 .
$$

Namely, each component of the vector-valued function $\Phi$ is a harmonic function.

An easy remark is that, for a given affine map $A: \mathbb{R}^{n} \rightarrow \mathbb{R}^{m}$ and a function $\Phi: X \rightarrow \mathbb{R}^{n}$, we have $\Delta(A \Phi)=A \Delta \Phi$, and hence if $\Phi$ is harmonic, then so is $A \Phi$.

We may easily show that the maximum principle holds for the discrete Laplacian, namely that a real-valued harmonic function which attains its maximum (or minimum) is constant. In particular, real-valued harmonic functions on a finite weighted graph are constant. We now establish the following fundamental theorem.

Theorem 2.3. Let $X$ be a finite weighted graph, and let $Y$ be a compact Riemannian manifold. Then each homotopy class of maps of $X$ into $Y$ contains at least one harmonic map.

Proof. Let $C$ be a homotopy class of maps of $X$ into $Y$. For each piecewise smooth map $\Phi \in C$, one can find a piecewise geodesic map $\Phi_{1} \in C$ with $E\left(\Phi_{1}\right) \leq E(\Phi)$. Indeed, it is enough to replace each $\Phi_{e}$ by the shortest geodesic homotopic to $\Phi_{e}$ relative to the end points. We denote by $C^{*}$ the set of piecewise geodesic maps in $C$, and on it we introduce the $C^{1}$-topology so that the functional $E$ is continuous on $C^{*}$. It is straightforward to see that, for every $a>0$, the sublevel set $\left\{\Phi \in C^{*} \mid E(\Phi) \leq a\right\}$ is compact. Therefore one can find a $\Phi_{0} \in C^{*}$ such that $E\left(\Phi_{0}\right) \leq E(\Phi)$ for every $\Phi \in C^{*}$. Since $\Phi_{0}$ is a critical map, it gives a desired harmonic map. 
The following theorem asserts the uniqueness of harmonic maps in a fixed homotopy class of maps into a flat torus.

Theorem 2.4. Let $g_{1}$ and $g_{2}$ be flat metrices on a torus $\mathcal{T}$, and let $X$ be a finite weighted graph. If two harmonic maps $\Phi_{1}: X \rightarrow\left(\mathcal{T}, g_{1}\right)$ and $\Phi_{2}: X \rightarrow\left(\mathcal{T}, g_{2}\right)$ are homotopic, then there exists an element $a \in \mathcal{T}$ such that $\Phi_{1}-\Phi_{2} \equiv a$.

Proof. Write $\mathcal{T}=\mathbb{R}^{n} / \Gamma$ with a lattice subgroup $\Gamma$ of $\mathbb{R}^{n}$, and let $\widetilde{\Phi}_{i}: \widetilde{X} \rightarrow \mathbb{R}^{n}$ be a lift of the harmonic map $\Phi_{i}$ to the universal covering graph $\widetilde{X}=(\widetilde{V}, \widetilde{E})$ of $X$ for $i=1,2$. Denote by $\rho: \pi_{1}(X) \rightarrow \Gamma$ the homomorphism induced from the map $\Phi_{i}$. We find that

$$
\widetilde{\Phi}_{i}(\sigma \widetilde{x})=\widetilde{\Phi}_{i}(\widetilde{x})+\rho(\sigma)
$$

for $\widetilde{x} \in \widetilde{X}$ and $\sigma \in \pi_{1}(X)$, and hence $\widetilde{\Phi}_{1}-\widetilde{\Phi}_{2}$ is a $\pi_{1}(X)$-invariant function on $\widetilde{X}$. The function $\widetilde{\Phi}_{1}-\widetilde{\Phi}_{2}$ is harmonic because the condition for a map of $\widetilde{X}$ into $\mathbb{R}^{n}$ to be harmonic does not depend on the choice of flat metrices on $\mathbb{R}^{n}$. Therefore $\widetilde{\Phi}_{1}-\widetilde{\Phi}_{2}$ is a lift of a harmonic function on $X$, and hence we conclude, in view of the maximum principle, that there exists $\widetilde{a} \in \mathbb{R}^{n}$ such that $\widetilde{\Phi}_{1}-\widetilde{\Phi}_{2} \equiv \widetilde{a}$. This completes the proof.

Theorem 2.5. Let $Y$ be a compact Riemannian manifold with non-positive sectional curvature, and let $C$ be a homotopy class of maps from a finite weighted graph $X$ into $Y$. Then a map $\Phi \in C$ is harmonic if and only if $\Phi$ minimizes the energy functional restricted to $C$. Furthermore, if $Y$ has strictly negative sectional curvature, and if a map in $C$ induces a homomorphism of $\pi_{1}(X)$ into $\pi_{1}(Y)$ whose image is not cyclic, then $C$ contains a unique harmonic map.

Proof. Let $\Phi_{0}$ and $\Phi_{1}$ be harmonic maps in $C$. One may choose lifts $\widetilde{\Phi}_{0}, \widetilde{\Phi}_{1}: \widetilde{X} \rightarrow \widetilde{Y}$ of $\Phi_{0}, \Phi_{1}$ to the universal covering spaces, respectively, satisfying

$$
\widetilde{\Phi}_{i}(\sigma \widetilde{x})=\rho(\sigma) \widetilde{\Phi}_{i}(\widetilde{x}) \quad\left(\sigma \in \pi_{1}(X) ; \widetilde{x} \in \widetilde{X} ; i=0,1\right)
$$

with the same induced homomorphism $\rho: \pi_{1}(X) \rightarrow \pi_{1}(Y)$. Joining $\widetilde{\Phi}_{0}(\widetilde{x})$ and $\widetilde{\Phi}_{1}(\widetilde{x})$ by a unique geodesic in $\widetilde{Y}$, one may define a piecewise smooth map $\widetilde{\Phi}_{u}$ : $\widetilde{X} \rightarrow \widetilde{Y}$ for $0 \leq u \leq 1$. It is easy to check that $\widetilde{\Phi}_{u}(\sigma \widetilde{x})=\rho(\sigma) \widetilde{\Phi}_{u}(\widetilde{x})$, so that one has a piecewise smooth map $\Phi_{u}: X \rightarrow Y$ in $C$ whose lift is $\widetilde{\Phi}_{u}$. Applying the second variation formula, we observe that $d^{2} E\left(\Phi_{u}\right) / d u^{2} \geq 0$ for $D_{W_{e}} W_{e}=0$. Since

$$
\left.\frac{d E\left(\Phi_{u}\right)}{d u}\right|_{u=0}=\left.\frac{d E\left(\Phi_{u}\right)}{d u}\right|_{u=1}=0
$$

we find that $d E\left(\Phi_{u}\right) / d u \equiv 0$, and hence $E\left(\Phi_{u}\right) \equiv$ constant. This implies the first assertion.

For the second assertion, we first observe from the second variation formula and nonpositivity of the curvature that $D W_{e} / d t \equiv 0$ and $\left\langle R\left(W_{e}, \dot{\Phi}_{e}(0)\right) \dot{\Phi}_{e}(0), W_{e}\right\rangle \equiv$ 0 since $d^{2} E\left(\Phi_{u}\right) / d u^{2} \equiv 0$. From the assumption that the curvature is strictly negative, it follows that $W(x)=c \dot{\Phi}_{e}(0)$ with some constant $c$. Since the image of $\rho$ is not cyclic, there exists a vertex $x$ of $X$ such that the dimension of the subspace spanned by $\left\{\dot{\Phi}_{e}(0) \mid e \in E_{x}\right\}$ is greater than one. Indeed, if the dimension is one or zero for every vertex, then the image of $\Phi$ lies in a circle in $Y$ so that the image of $\rho$ is cyclic; this is a contradiction. Thus $W_{e} \equiv 0$ for $e \in E_{x}$, and hence for all $e \in E$ because $D W_{e} / d t \equiv 0$. This implies that $\Phi_{1}=\Phi_{2}$, as desired. 
Given an $n$-dimensional compact smooth manifold $N$, we consider a family $\mathcal{G}$ of Riemannian metrices on $N$ with a fixed volume. Take a homotopy class $C$ of maps of a finite weighted graph $X$ into $N$, and consider the function $E=E(\Phi, g)$ on $C \times \mathcal{G}$. Closely related to the subject in this paper is the following general problem. Does the function E attain its minimum at some pair $\left(\Phi_{0}, g_{0}\right)$ ? Actually the first part of Theorem 2 says that this is the case when $N$ is a torus, $\mathcal{G}$ is the family of flat metrics on $N$, and maps in $C$ induce a surjective homomorphism of $\pi_{1}(X)$ onto $\pi_{1}(N)$. It is rather easy, in this special case, to show the existence of such a pair. The core of our problem is to detect $\left(\Phi_{0}, g_{0}\right)$ in an explicit way.

It is interesting to investigate the case of a surface $N$ with the family $\mathcal{G}$ of constant negatively curved metrices, which is regarded as a non-Euclidean analogue of our problem in dimension two.

Remark. Recently much attention has been paid to harmonic maps between singular metric spaces including graphs (see [4, [5], [6], [10, [12] for instance). Actually the existence of energy minimizing harmonic maps of a finite weighted graph into a fixed flat torus is a very special case of the results in more general situations [6].

We should also remark that in [3], Ejiri studied the index of compact minimal surfaces in tori by looking at the variation of flat structures of tori.

\section{Reduction to A GeOmetric problem}

We shall explain briefly how Theorem 1 is reduced to the problem in terms of flat tori and weighted graphs as stated in Theorem 2 .

With a system of springs in $\mathbb{R}^{n}$, one may always associate a graph in such a way that the springs correspond to unoriented edges and the points of masses correspond to vertices, where the incidence of edges and vertices is defined in a natural manner. The two atoms connected by a spring are supposed to interact each other by the dynamical law of a harmonic oscillator. Regarding the masses and the force constants as weights of vertices and edges respectively, we have a weighted graph. Furthermore, joining two adjacent vertices by the line segment, we have a piecewise geodesic map $\Phi$ from the graph into the Euclidean space, where $\Phi(x)$ designates the position of a mass $x$. The force acting on the mass $x$ generated by the spring $e$ with $o(e)=x$ is given by $m_{E}(e)(\Phi(t(e))-\Phi(o(e)))$, where $m_{E}(e)$ is the force constant of the spring $e$. By the principle of superposition, we conclude that the total force acting on the mass $x$ is given by

$$
\sum_{e \in E_{x}} m_{E}(e)(\Phi(t(e))-\Phi(o(e))) .
$$

Therefore, in view of (14), the internal forces in the system of springs is in equilibrium if and only if $\Phi$ is harmonic. For a finite system of springs, $E(\Phi)$ coincides with the potential energy of the system. It is worth mentioning that the vibration of the system is described by Newton's dynamical equation

$$
\frac{d^{2} \Phi}{d t^{2}}=\Delta \Phi
$$

which is regarded as a discrete analogue of the wave equation.

Consider the system of springs corresponding to a crystal in $\mathbb{R}^{n}$, which need not have the equilibrium configuration. The additive group $\Gamma$ of translations acting on the system is a lattice group, known as the Bravais lattice, namely a group 
consisting of vectors $\mathbf{x}$ represented as integral linear combinations of $n$ linearly independent vectors $\mathbf{v}_{1}, \mathbf{v}_{2}, \cdots, \mathbf{v}_{n}$ :

$$
\Gamma=\left\{\mathbf{x}=\sum_{i=1}^{n} k_{i} \mathbf{v}_{i} \mid k_{i} \text { integers }\right\} .
$$

As a primitive unit cell of the crystal, we may take a fundamental parallelotope $P$ in the space for the action of $\Gamma$, that is,

$$
P=\left\{\mathbf{x}=\sum_{i=1}^{n} a_{i} \mathbf{v}_{i} \mid 0 \leq a_{i}<1\right\} .
$$

We identify $P$ with the torus $T_{\Gamma}=\mathbb{R}^{n} / \Gamma$. The torus $\mathcal{T}_{\Gamma}$ has the flat metric induced from the one on the Euclidean space $\mathbb{R}^{n}$. The volume of the primitive unit cell is nothing but the volume $\operatorname{vol}\left(\mathcal{T}_{\Gamma}\right)$ of the flat torus $\mathcal{T}_{\Gamma}$ as a Riemannian manifold. It should be noted here that

$$
\operatorname{vol}\left(\mathcal{T}_{\Gamma}\right)=\operatorname{vol}(P)=\left(\operatorname{det}\left(\left\langle\mathbf{v}_{i}, \mathbf{v}_{j}\right\rangle\right)\right)^{1 / 2} .
$$

On the other hand, we identify two masses in the system if one of them is obtained by translation in $\Gamma$ from the other. We also identify two springs in the same way. We thus have a finite system of springs, which is realized in the flat torus $\mathcal{T}_{\Gamma}$.

Applying the general recipe above to the system associated with a crystal, we obtain two weighted graphs, one of which, denoted by $X$, corresponds to the crystal itself and the other, denoted by $X_{0}$, corresponds to the finite system realized in the flat torus. The piecewise geodesic map $\Phi$ gives a configuration of the crystal. The piecewise geodesic map $\Phi_{0}$ of $X_{0}$ into $\mathcal{T}_{\Gamma}$ is the one obtained by projecting down the map $\Phi$ to $X_{0}$. From the construction, it follows that $\Phi_{0}$ is harmonic if and only if $\Phi$ is harmonic. Therefore the internal forces in the crystal are in equilibrium if and only if the map $\Phi_{0}$ is harmonic. We define the potential energy per primitive unit cell as the energy of $\Phi_{0}$.

The graph $X$ is called the crystal lattice. The lattice group $\Gamma$ acts on $X$ as a graph automorphism. In the context of graph theory, $X_{0}$ is the quotient graph of $X$ by the action of $\Gamma$. From now on, we consider $\Gamma$ as an abstract group acting on $X$. When a configuration of the crystal varies, $\mathcal{T}_{\Gamma}$, the primitive unit cell, changes, although its topological type does not. In other words, according as the piecewise geodesic map $\Phi_{0}$ varies, the flat metirc $g$ on the torus changes. Therefore we paraphrase our problem with this terminology in the following way. Fix a topological torus $\mathcal{T}$ and a homotopy class $C$ of maps from $X_{0}$ to $\mathcal{T}$, and let $v_{0}>0$. An equilibrium configuration of the crystal is a lift of a pair $\left(\Phi_{0}, g_{0}\right)$ which minimizes the energy $E=E(\Phi, g)$ under the condition that $\Phi_{0} \in C$ and $\operatorname{vol}(\mathcal{T}, g)=v_{0}$, where, from its nature, the homotopy class $C$ is supposed to satisfy the condition that a map in $C$ induces the surjective homomorphism of $\pi_{1}\left(X_{0}\right)$ onto $\pi_{1}(\mathcal{T})$. We are thus led to the setup described in Theorem 2 It should be noted that the masses of atoms do not play any role as far as the static nature of crystals is concerned.

We shall illustrate the above discussion by taking a look at the diamond crystal. For simplicity, the weights of vertices and edges are taken to be one. Let $\mathbf{e}_{1}, \mathbf{e}_{2}, \mathbf{e}_{3}$ be the standard basis of $\mathbb{R}^{3}$. We consider the lattice group $\Gamma$ generated by the basis $\mathbf{v}_{1}=\mathbf{e}_{2}+\mathbf{e}_{3}=(0,1,1), \mathbf{v}_{2}=\mathbf{e}_{3}+\mathbf{e}_{1}=(1,0,1), \mathbf{v}_{3}=\mathbf{e}_{1}+\mathbf{e}_{2}=(1,1,0) . \Gamma$ is what we call the face centered cubic lattice group. It is easy to check that $\operatorname{vol}\left(\mathcal{T}_{\Gamma}\right)=2$. 

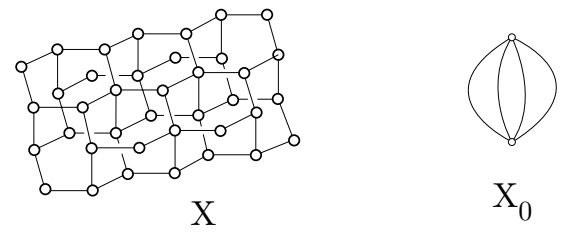

FIgURE 1. The diamond lattice and its quotient graph

The sites of carbon atoms are divided into two kinds:

$$
\begin{aligned}
A & =\Gamma=\left\{\left(m_{2}+m_{3}, m_{3}+m_{1}, m_{1}+m_{2}\right) \mid m_{i} \in \mathbb{Z}\right\}, \\
B & =(1 / 2,1 / 2,1 / 2)+\Gamma \\
& =\left\{\left(n_{2}+n_{3}+1 / 2, n_{3}+n_{1}+1 / 2, n_{1}+n_{2}+1 / 2\right) \mid n_{i} \in \mathbb{Z}\right\} .
\end{aligned}
$$

Two atoms at sites $\left(m_{2}+m_{3}, m_{3}+m_{1}, m_{1}+m_{2}\right),\left(n_{2}+n_{3}+1 / 2, n_{3}+n_{1}+1 / 2, n_{1}+\right.$ $\left.n_{2}+1 / 2\right)$ are to be binded by an edge if and only if one of the followings holds:

$$
\begin{array}{lll}
m_{1}=n_{1}, & m_{2}=n_{2}, & m_{3}=n_{3}, \\
m_{1}=n_{1}+1, & m_{2}=n_{2}, & m_{3}=n_{3}, \\
m_{1}=n_{1}, & m_{2}=n_{2}+1, & m_{3}=n_{3}, \\
m_{1}=n_{1}, & m_{2}=n_{2}, & m_{3}=n_{3}+1,
\end{array}
$$

In this way, we have a crystal lattice $X$ for a diamond, which we call the diamond lattice. It should be noted that the origin $\mathbf{o}$ is in $A$, and the vertices in the nearest neighbor of $\mathbf{o}$ are

$$
\begin{aligned}
& \mathbf{a}_{1}=(1 / 2,1 / 2,1 / 2), \\
& \mathbf{a}_{2}=(1 / 2,-1 / 2,-1 / 2), \\
& \mathbf{a}_{3}=(-1 / 2,1 / 2,-1 / 2), \\
& \mathbf{a}_{4}=(-1 / 2,-1 / 2,1 / 2) .
\end{aligned}
$$

We observe that $\mathbf{a}_{1}, \mathbf{a}_{2}, \mathbf{a}_{3}, \mathbf{a}_{4}$ form the regular tetrahedron whose center of mass is o. It is also easily checked that the quotient graph $X_{0}$ is the graph with two vertices joined by 3 multiple edges. The potential energy per primitive unit cell is equal to 3 . We shall show in $\$ 5$ that this configuration is the equilibrium configuration.

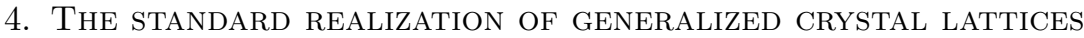

We shall set up the class of graphs which generalizes the notion of crystal lattices.

A locally finite connected weighted graph $X=(V, E)$ with weight functions $m_{V}$ and $m_{E}$ is said to be a generalized crystal lattice or $\Gamma$-crystal lattice if the following conditions are satisfied.

1. There exists a free abelian automorphism group $\Gamma$ of $X$ which acts freely on the set $V$ and the set of unoriented edges.

2. The weight functions are $\Gamma$-invariant.

3. The quotient graph $\Gamma \backslash X=X_{0}$ is finite.

In other words, $X$ is an abelian covering graph of a finite weighted graph $X_{0}$ with the covering transformation group $\Gamma$, and the weight functions on $X$ are the lifts of those on $X_{0}$.

The weighted graph associated with a crystal which we considered in $\$ 3$ is a $\Gamma$-crystal lattice with the Bravais lattice $\Gamma$. 
Let $X$ be a $\Gamma$-crystal lattice with the quotient graph $X_{0}=\Gamma \backslash X$. Let $\varphi: X \rightarrow X_{0}$ be the covering map and let $\varphi^{a b}: X_{0}^{a b} \rightarrow X_{0}$ be the maximal abelian covering map with the covering transformation group $H_{1}\left(X_{0}, \mathbb{Z}\right)$, the 1-homology group with coefficients in $\mathbb{Z}$. Then there exists a covering map $\varphi_{1}: X_{0}^{a b} \rightarrow X$ such that $\varphi \circ \varphi_{1}=\varphi^{a b}$.

We shall introduce several inner product spaces over $\mathbb{R}$ associated with the finite weighted graph $X_{0}$. We adopt the following convention throughout this section. Given an inner product space $\mathcal{V}$, the dual vector space and quotient vector spaces of $\mathcal{V}$ are considered as inner product spaces with the canonical inner products obtained from that of $\mathcal{V}$.

We denote by $C^{i}\left(X_{0}, \mathbb{R}\right)$ the $i$-th cochain group $(i=0,1)$ of the finite graph $X_{0}=\left(V_{0}, E_{0}\right)$ with the following inner product:

$$
\begin{aligned}
& \left\langle f_{1}, f_{2}\right\rangle=\sum_{x \in V_{0}} m_{V_{0}}(x) f_{1}(x) f_{2}(x) \quad\left(f_{1}, f_{2} \in C^{0}\left(X_{0}, \mathbb{R}\right)\right) \\
& \left\langle\omega_{1}, \omega_{2}\right\rangle=\frac{1}{2} \sum_{e \in E_{0}} m_{E_{0}}(e) \omega_{1}(e) \omega_{2}(e) \quad\left(\omega_{1}, \omega_{2} \in C^{1}\left(X_{0}, \mathbb{R}\right)\right) .
\end{aligned}
$$

The adjoint operator $\delta: C^{1}\left(X_{0}, \mathbb{R}\right) \rightarrow C^{0}\left(X_{0}, \mathbb{R}\right)$ of the coboundary operator $d:$ $C^{0}\left(X_{0}, \mathbb{R}\right) \rightarrow C^{1}\left(X_{0}, \mathbb{R}\right)$ is given by

$$
(\delta \omega)(x)=-m_{V_{0}}(x)^{-1} \sum_{e \in\left(E_{0}\right)_{x}} \omega(e) m_{E_{0}}(e) .
$$

The operators $d$ and $\delta$ extend to vector-valued cochains in a natural manner.

It should be noted that $\Delta=-\delta d$. Since (Image $d)^{\perp}=\operatorname{Ker} \delta$, we may identify the 1-cohomology group $H^{1}\left(X_{0}, \mathbb{R}\right)$, which is the quotient inner product space $C^{1}\left(X_{0}, \mathbb{R}\right) /$ Image $d$, with the subspace $\operatorname{Ker} \delta$ of $C^{1}\left(X_{0}, \mathbb{R}\right)$, the space of "harmonic 1-forms". Next we consider the $i$-th chain group $C_{i}\left(X_{0}, \mathbb{R}\right)$, which is considered to be the dual inner product space of $C^{i}\left(X_{0}, \mathbb{R}\right)$. More explicitly, the inner product . on $C_{1}\left(X_{0}, \mathbb{R}\right)$ is given by

$$
e \cdot e^{\prime}= \begin{cases}m_{E_{0}}(e)^{-1}, & e=e^{\prime} \\ -m_{E_{0}}(e)^{-1}, & e=\bar{e}^{\prime} \\ 0, & \text { otherwise }\end{cases}
$$

Let $X$ be a $\Gamma$-crystal lattice with the quotient graph $X_{0}$. We extend the homomorphism $\rho: H_{1}\left(X_{0}, \mathbb{Z}\right) \rightarrow \Gamma$ associated with the covering map $\varphi: X \rightarrow X_{0}$ to the linear map $\rho_{\mathbb{R}}: H_{1}\left(X_{0}, \mathbb{R}\right)=H_{1}\left(X_{0}, \mathbb{Z}\right) \otimes \mathbb{R} \rightarrow \Gamma \otimes \mathbb{R}$. Note that $\rho_{\mathbb{R}}\left(H_{1}\left(X_{0}, \mathbb{Z}\right)\right)=\Gamma$. We regard $\Gamma \otimes \mathbb{R}$ as a quotient inner product space of $H_{1}\left(X_{0}, \mathbb{R}\right)$ and denote the inner product by $\langle,\rangle_{\text {alb }}$. The flat metric on the torus $\Gamma \otimes \mathbb{R} / \Gamma$ induced from $\langle,\rangle_{a l b}$ is called the Albanese metric and is denoted by $g_{a l b}$. We write $A l b^{\Gamma}$ for $\left(\Gamma \otimes \mathbb{R} / \Gamma, g_{a l b}\right)$, and call it the $\Gamma$-Albanese torus. In the case of the maximal abelian covering graph,

$$
A l b^{\Gamma}=\operatorname{Alb}\left(X_{0}\right)=H_{1}\left(X_{0}, \mathbb{R}\right) / H_{1}\left(X_{0}, \mathbb{Z}\right)
$$

The transpose $\rho_{\mathbb{R}}^{*}$ of $\rho_{\mathbb{R}}$ is an injective map of $\operatorname{Hom}(\Gamma, \mathbb{R})=(\Gamma \otimes \mathbb{R})^{*}$ into $H^{1}\left(X_{0}, \mathbb{R}\right)$. From now on, we consider $\operatorname{Hom}(\Gamma, \mathbb{R})$ as a subspace of $H^{1}\left(X_{0}, \mathbb{R}\right)$ via $\rho_{\mathbb{R}}^{*}$. 
We shall define a harmonic map $\Phi^{\Gamma}: X_{0} \rightarrow A l b^{\Gamma}$. For this, we first define $\widetilde{\Phi}^{\Gamma}: V \rightarrow \Gamma \otimes \mathbb{R}$ by

$$
\widetilde{\Phi}^{\Gamma}(x)(\omega)=\int_{x_{0}}^{x} \tilde{\omega},
$$

where $\tilde{\omega}$ is the lift of a harmonic 1-form $\omega \in \operatorname{Hom}(\Gamma, \mathbb{R}) \subset H^{1}\left(X_{0}, \mathbb{R}\right)$ and the integral in the right hand side is defined, for a path $c=\left(e_{1}, \ldots, e_{l}\right)$ with $o(c)=$ $x_{0}, t(c)=x$, by

$$
\int_{x_{0}}^{x} \tilde{\omega}=\tilde{\omega}\left(e_{1}\right)+\cdots+\tilde{\omega}\left(e_{l}\right) .
$$

It should be noted that $\widetilde{\Phi}^{\Gamma}(x)(\omega)$ does not depend on the choice of a path $c$. Next we put, for each $e \in E$,

$$
\widetilde{\Phi}_{e}^{\Gamma}(t)=\widetilde{\Phi}^{\Gamma}(e, t)=(1-t) \widetilde{\Phi}^{\Gamma}(o(e))+t \widetilde{\Phi}^{\Gamma}(t(e)) .
$$

In this way, we have a piecewise geodesic map $\widetilde{\Phi}^{\Gamma}: X \rightarrow \Gamma \otimes \mathbb{R}$. Note that, for $\sigma \in \Gamma$,

$$
\widetilde{\Phi}^{\Gamma}(\sigma x)=\widetilde{\Phi}^{\Gamma}(x)+\sigma,
$$

since $\int_{x_{0}}^{\sigma x_{0}} \widetilde{\omega}=\omega(\sigma)$, and for every $\omega \in \operatorname{Hom}(\Gamma, \mathbb{R})$,

$$
\sum_{e \in E_{x}} m_{E}(e)\left(\widetilde{\Phi}^{\Gamma}(t(e))-\widetilde{\Phi}^{\Gamma}(o(e))\right)(\widetilde{\omega})=\sum_{e \in E_{x}} m_{E}(e) \widetilde{\omega}(e)=0 .
$$

We thus obtain a harmonic map $\Phi^{\Gamma}: X_{0} \rightarrow A l b^{\Gamma}$.

The map $\Phi^{\Gamma}$ is an analogue of the Albanese maps, which were originally defined in algebraic geometry and Riemannian geometry (see [9]). The image $\widetilde{\Phi}^{\Gamma}$ is called the standard realization of the generalized crystal lattice $X$.

Let $X$ be a $\Gamma$-crystal lattice with $X_{0}$ as its quotient graph, and let $\tau: \Gamma \rightarrow \Gamma_{1}$ be a surjective homomorphism onto a free abelian group $\Gamma_{1}$. The quotient graph $\operatorname{Ker} \tau \backslash X$ is a $\Gamma_{1}$-crystal graph with $X_{0}$ as its quotient graph. The inner product space $\Gamma_{1} \otimes \mathbb{R}$ is a quotient inner product space of $\Gamma \otimes \mathbb{R}$ via the linear map $\tau_{\mathbb{R}}: \Gamma \otimes \mathbb{R} \rightarrow$ $\Gamma_{1} \otimes \mathbb{R}$ induced from $\tau$. We thus obtain a Riemannian submersion $\tau_{*}: A l b^{\Gamma} \rightarrow A l b^{\Gamma_{1}}$ in a natural manner. We may easily check that $\tau_{\mathbb{R}} \circ \widetilde{\Phi}^{\Gamma}=\widetilde{\Phi}^{\Gamma_{1}} \circ \varphi_{1}$ and $\tau_{*} \circ \Phi^{\Gamma}=\Phi^{\Gamma_{1}}$, where $\varphi_{1}: X \rightarrow X_{1}$ denotes the canonical projection. In particular, applying this discussion to the homomorphism $\rho: H_{1}\left(X_{0}, \mathbb{Z}\right) \rightarrow \Gamma$ associated with the covering map $\varphi: X \rightarrow X_{0}$, we find that the standard realization of $X$ in $\Gamma \otimes \mathbb{R}$ coincides with the image of the standard realization for the maximal abelian covering $X_{0}^{a b}$ in $H_{1}\left(X_{0}, \mathbb{R}\right)$ by the orthogonal projection $\rho_{\mathbb{R}}: H_{1}\left(X_{0}, \mathbb{R}\right) \rightarrow \Gamma \otimes \mathbb{R}$.

Next, we let $\Gamma_{1}$ be a subgroup of $\Gamma$ with finite index $\ell$. A $\Gamma$-crystal lattice $X$ is also considered as a $\Gamma_{1}$-crystal lattice with the finite quotient graph $X_{1}=\Gamma_{1} \backslash X$. The covering map $\varphi: X \rightarrow X_{0}$ is factored as

$$
X \stackrel{\varphi_{1}}{\longrightarrow} X_{1} \stackrel{\varphi_{0}}{\longrightarrow} X_{0},
$$

where $\varphi_{0}$ is an $\ell$-fold covering map. Let $\iota: \Gamma_{1} \rightarrow \Gamma$ be the inclusion map. We observe that the linear map $\iota_{\mathbb{R}}: \Gamma_{1} \otimes \mathbb{R} \rightarrow \Gamma \otimes \mathbb{R}$ satisfies

$$
\left\langle\iota_{\mathbb{R}}(\mathbf{u}), \iota_{\mathbb{R}}(\mathbf{v})\right\rangle_{\text {alb }}=\ell\langle\mathbf{u}, \mathbf{v}\rangle_{\text {alb }},
$$

and induces a homothetic $\ell$-fold covering map $\iota_{*}: A l b_{1}^{\Gamma} \rightarrow A l b^{\Gamma}$. From the construction of Albanese maps, we find that $\iota_{\mathbb{R}} \circ \widetilde{\Phi}^{\Gamma_{1}}=\widetilde{\Phi}^{\Gamma}$ and $\iota_{*} \circ \Phi^{\Gamma_{1}}=\Phi^{\Gamma} \circ \varphi_{0}$. 
This implies that the standard realization of the crystal lattice $X$ up to homothety depends only on the commensurable class of groups $\Gamma$. It is easily checked that $\operatorname{vol}\left(A l b^{\Gamma}\right)=\ell^{k / 2-1} \operatorname{vol}\left(A l b_{1}^{\Gamma}\right)$, where $k=\operatorname{rank} \Gamma=\operatorname{rank} \Gamma_{1}$.

We shall now see that the Albanese map $\Phi^{\Gamma}: X_{0} \rightarrow A l b^{\Gamma}$ is characterized as a map with minimal energy among piecewise smooth maps, which is homotopic to $\Phi^{\Gamma}$, of $X_{0}$ into the torus $\Gamma \otimes \mathbb{R} / \Gamma$ with a flat metric whose volume is the same as $\operatorname{vol}\left(A l b^{\Gamma}\right)$.

Theorem 4.1. Let $g$ be a flat metric on the torus $\mathcal{T}=\Gamma \otimes \mathbb{R} / \Gamma$ with the same volume as $g_{a l b}$, and let $\Phi$ be a piecewise smooth map from $X_{0}$ to $(\mathcal{T}, g)$. If $\Phi$ is homotopic to $\Phi^{\Gamma}$, then

$$
E\left(\Phi^{\Gamma}, g_{a l b}\right) \leq E(\Phi, g)
$$

The equality holds if and only if $(\mathcal{T}, g)=\left(\mathcal{T}, g_{\text {alb }}\right)=A l b^{\Gamma}$ and $\Phi$ coincides with $\Phi^{\Gamma}$ up to a translation.

We preface the proof with two lemmas.

A path in a graph is a sequence $c=\left(e_{1}, \ldots, e_{l}\right)$ with $t\left(e_{i}\right)=o\left(e_{i+1}\right), i=1, \ldots$, $l-1$. We put $o(c)=o\left(e_{1}\right), t(c)=t\left(e_{l}\right)$. When $o(c)=t(c)$, the path $c$ is said to be closed. A closed path without self-intersections is called a circuit.

Given a circuit $c=\left(e_{1}, \ldots, e_{l}\right)$ in $X_{0}$, we define a cochain $\omega_{c}$ by

$$
\omega_{c}(e)= \begin{cases}m_{E_{0}}\left(e_{i}\right)^{-1}, & e=e_{i}, \\ -m_{E_{0}}\left(e_{i}\right)^{-1}, & \bar{e}=e_{i}, \\ 0, & \text { otherwise }\end{cases}
$$

We observe that $\omega_{c}$ is a harmonic 1 -form and

$$
\left\langle\omega_{c_{1}}, \omega_{c_{2}}\right\rangle=\omega_{c_{1}}\left(c_{2}\right)=c_{1} \cdot c_{2} .
$$

An orientation of $X_{0}$ is given by a subset $E_{0}^{o}$ of $E_{0}$ such that $E_{0}=E_{0}^{o} \amalg \overline{E_{0}^{o}}$, where $\overline{E_{0}^{o}}=\left\{\bar{e} \mid e \in E_{0}^{o}\right\}$. Let $N$ be the number of vertices and $M$ the number of unoriented edges of $X_{0}$.

Lemma 4.2. There exists a $\mathbb{Z}$-basis $c_{1}, \ldots, c_{n}$ of the 1 -homology group $H_{1}\left(X_{0}, \mathbb{Z}\right)$ $\left(n=\operatorname{rank} H_{1}\left(X_{0}, \mathbb{Z}\right)\right)$ consisting of circuits.

Proof. Take a maximal (spanning) tree $T=\left(V_{T}, E_{T}\right)$ of $X_{0}$, a maximal element among subtrees ordered by inclusion. The tree $T$ contains all vertices of $X_{0}$, and the number of the unoriented edges not contained in $T$ is equal to $n$. Give an orientation $E_{0}^{o}=\left\{e_{1}, \ldots, e_{M}\right\}$ to the graph $X_{0}$ such that

$$
\begin{array}{r}
e_{1}, \ldots, e_{n} \notin E_{T}, \\
e_{n+1}, \ldots, e_{M} \in E_{T} .
\end{array}
$$

For $i$ with $1 \leq i \leq n$, take the minimal path $c_{i}^{\prime}$ in $T$ such that $o\left(c_{i}^{\prime}\right)=t\left(e_{i}\right), t\left(c_{i}^{\prime}\right)=$ $o\left(e_{i}\right)$, and put $c_{i}=e_{i} c_{i}^{\prime}$. We easily find that the circuits $c_{1}, \ldots, c_{n}$ form a basis of $H_{1}\left(X_{0}, \mathbb{R}\right)$.

Let $c_{1}, \ldots, c_{n}$ be a $\mathbb{Z}$-basis of the 1 -homology group $H_{1}\left(X_{0}, \mathbb{Z}\right)$ consisting of circuits, and let $u_{1}, \ldots, u_{n} \in H^{1}\left(X_{0}, \mathbb{R}\right)$ be the dual basis, i.e. $u_{i}\left(c_{j}\right)=\delta_{i j}$. Put 
$\omega_{i}=\omega_{c_{i}}$. Then $\omega_{1}, \ldots, \omega_{n}$ form an $\mathbb{R}$-basis of $H^{1}\left(X_{0}, \mathbb{R}\right)$. Therefore one can find an $n \times n$ matrix $U=\left(u_{i j}\right)$ such that

$$
u_{i}=\sum_{j=1}^{n} u_{i j} \omega_{j}
$$

Choose an orientation $E_{0}^{o}=\left\{e_{1}, \ldots e_{M}\right\}$ of $X_{0}$ and put $W=\operatorname{diag}\left(\mu_{1}, \ldots, \mu_{M}\right)$ with $\mu_{i}=m_{E}\left(e_{i}\right)^{-1}$. Writing $c_{i}=\sum_{l=1}^{M} f_{i l} e_{l}$, we define an $n \times M$ matrix $F=\left(f_{i l}\right)$. It is easy to check

Lemma 4.3. Put $\Lambda=\left(c_{i} \cdot c_{j}\right)$. Then

$$
\begin{gathered}
\Lambda=U^{-1} \\
\Lambda=F W F^{t} .
\end{gathered}
$$

Proof. The first equality follows from

$$
\delta_{i j}=u_{i}\left(c_{j}\right)=\sum_{h=1}^{n} u_{i h} \omega_{h}\left(c_{j}\right)=\sum_{h=1}^{n} u_{i h} \Lambda_{h j} .
$$

The second one is checked as

$$
\begin{aligned}
c_{i} \cdot c_{j} & =\left(\sum_{l=1}^{M} f_{i l} e_{l}\right) \cdot\left(\sum_{m=1}^{M} f_{j m} e_{m}\right) \\
& =\sum_{l, m=1}^{M} f_{i l} f_{j m} e_{l} \cdot e_{m}=\sum_{l=1}^{M} f_{i l} \mu_{l} f_{j l} .
\end{aligned}
$$

Now we are ready to prove Theorem 4.1 .

Proof. We may assume $\Phi$ is harmonic because a harmonic map into a torus with a fixed flat metric is energy minimizing (Theorem 2.5). Owing to Theorem 2.4, $\Phi$ is equal to $\Phi^{\Gamma}$ up to a translation as a map to $\mathcal{T}=\Gamma \otimes \mathbb{R} / \Gamma$, and we only have to find a flat metric $g$ on $\mathcal{T}$ with a fixed volume which minimizes the energy $E\left(\Phi^{\Gamma}, g\right)$. We shall show that such a metric is homothetic to the Albanese metric. Without loss of generality, we may assume that $\operatorname{vol}(\mathcal{T}, g)=\operatorname{vol}\left(\mathcal{T}, g_{\text {alb }}\right)$.

Take a $\mathbb{Z}$-basis $\sigma_{1}, \ldots, \sigma_{k}$ of $\Gamma$, and let $v_{1}, \ldots, v_{k} \in \operatorname{Hom}(\Gamma, \mathbb{R})$ be the dual basis of $\sigma_{1}, \ldots, \sigma_{k}$, where $k=\operatorname{rank} \Gamma$. Write

$$
c_{i}^{\prime}=\rho_{\mathbb{R}}\left(c_{i}\right)=\sum_{j=1}^{k} r_{j i} \sigma_{j} \quad\left(r_{j i} \in \mathbb{Z} ; i=1, \ldots, n\right) .
$$

Then we have $v_{j}=\rho_{\mathbb{R}}^{*}\left(v_{j}\right)=\sum_{i=1}^{n} r_{j i} u_{i}$. Put $S=\left(\left\langle v_{i}, v_{j}\right\rangle\right)=R U R^{t}$. Note that $S^{-1}=\left\langle\sigma_{i}, \sigma_{j}\right\rangle_{\text {alb }}$.

Regard $\widetilde{\Phi}\left(=\widetilde{\Phi}^{\Gamma}\right)$ as a 0 -cochain of $X$ with values in the vector space $\Gamma \otimes \mathbb{R}$. It is obvious that $d \widetilde{\Phi} \in C^{1}(X, \Gamma \otimes \mathbb{R})$ satisfies $d \widetilde{\Phi}(\sigma e)=d \widetilde{\Phi}(e)$ for $e \in E$ and $\sigma \in \Gamma$. Therefore $d \widetilde{\Phi}$ is identified with a 1 -cochain of $X_{0}$ with values in $\Gamma \otimes \mathbb{R}$.

Put

$$
\mathbf{v}_{i}=d \widetilde{\Phi}\left(e_{i}\right) \quad \text { for } i=1, \ldots, M
$$


Now, for $\omega \in \operatorname{Hom}(\Gamma, \mathbb{R}) \subset H^{1}\left(X_{0}, \mathbb{R}\right)$, we have

$$
\begin{aligned}
\omega\left(e_{i}\right) & =\sum_{j}\left\langle\omega, u_{j}\right\rangle u_{j}\left(e_{i}\right)=\sum_{j} \omega\left(c_{j}\right) u_{j}\left(e_{i}\right)=\sum_{j}\left(\rho_{\mathbb{R}}^{*} \omega\right)\left(c_{j}\right) u_{j}\left(e_{i}\right) \\
& =\sum_{j} \omega\left(\rho_{\mathbb{R}}\left(c_{j}\right)\right) u_{j}\left(e_{i}\right)=\sum_{j} \omega\left(c_{j}^{\prime}\right) u_{j}\left(e_{i}\right),
\end{aligned}
$$

and $\mathbf{v}_{i}(\omega)=\omega\left(e_{i}\right)$. Therefore we have

$$
\begin{aligned}
\mathbf{v}_{i} & =\sum_{j=1}^{n} u_{j}\left(e_{i}\right) c_{j}^{\prime}=\sum_{j=1}^{n} \sum_{m=1}^{n} u_{j m} \omega_{m}\left(e_{i}\right) c_{j}^{\prime} \\
& =\sum_{j=1}^{n} \sum_{m=1}^{n} u_{j m} f_{m i} \mu_{i} c_{j}^{\prime}=\sum_{j=1}^{n} \sum_{m=1}^{n} \sum_{l=1}^{k} u_{j m} f_{m i} \mu_{i} r_{l j} \sigma_{l} \\
& =\sum_{l=1}^{k}\left(W F^{t} U R^{t}\right)_{i l} \sigma_{l} .
\end{aligned}
$$

We put $A=\left(\left\langle\sigma_{i}, \sigma_{j}\right\rangle_{g}\right)$. Since

$$
\begin{aligned}
\operatorname{vol}\left(\Gamma \otimes \mathbb{R} / \Gamma, g_{a l b}\right) & =(\operatorname{det} S)^{-1 / 2} \\
\operatorname{vol}(\Gamma \otimes \mathbb{R} / \Gamma, g) & =(\operatorname{det} A)^{1 / 2}
\end{aligned}
$$

we have $\operatorname{det}(S A)=1$.

The energy $E\left(\Phi^{\Gamma}, g\right)$ is computed in the following way:

$$
\begin{aligned}
E\left(\Phi^{\Gamma}, g\right) & =\sum_{i=1}^{M} m_{E}\left(e_{i}\right)\left\|\mathbf{v}_{i}\right\|_{g}^{2}=\sum_{i=1}^{M} \sum_{j, h=1}^{k} \mu_{i}^{-1}\left(W F^{t} U R^{t}\right)_{i j}\left(W F^{t} U R^{t}\right)_{i h} A_{j h} \\
& =\operatorname{tr}\left(F^{t} U R^{t} A\left(W F^{t} U R^{t}\right)^{t}\right)=\operatorname{tr}\left(R U F W F^{t} U R^{t} A\right) \\
& =\operatorname{tr}\left(R U \Lambda U R^{t} A\right)=\operatorname{tr}\left(R U R^{t} A\right)=\operatorname{tr}(S A) .
\end{aligned}
$$

Let $\sqrt{S}$ be the positive square root of the positive definite symmetric matrix $S$. Then we have

$$
\operatorname{tr}(S A)=\operatorname{tr}(\sqrt{S} A \sqrt{S}) \geq k \operatorname{det}(\sqrt{S} A \sqrt{S})^{1 / k}=k \operatorname{det}(S A)^{1 / k}=k .
$$

The equality holds if and ony if $S A=I$, or equivalently

$$
\left\langle\sigma_{i}, \sigma_{j}\right\rangle_{g}=\left\langle\sigma_{i}, \sigma_{j}\right\rangle_{a l b}
$$

i.e. $g=g_{a l b}$.

Since $E\left(\Phi^{\Gamma}, c g\right)=c E\left(\Phi^{\Gamma}, g\right)$ and $\operatorname{vol}(c g)=c^{k / 2} \operatorname{vol}(g)$ for $c>0$, the above proof leads us to the following

Theorem 4.4. Let $X$ be a $k$-dimensional crystal lattice with the Bravais lattice group $\Gamma$, and let $\mathcal{E}_{0}$ be the potential energy per primitive unit cell with volume $v_{0}$. Then

$$
\mathcal{E}_{0} v_{0}^{-2 / k} \geq k \operatorname{vol}\left(A l b^{\Gamma}\right)^{-2 / k}
$$

The equality holds if and only if the configuration of $X$ is in equilibrium. 
So far we have explained how the equilibrium configurations of crystal lattices are described in terms of the Albanese maps into the Albanese tori. We are now in position to prove Theorem 2 which is actually a disguised form of Theorem 4.1 .

Let $C$ be a homotopy class of maps from a finite weighted graph $X_{0}$ into a torus $(\mathcal{T}, g)$ which induces a surjective homomorphism $\eta: \pi_{1}\left(X_{0}\right) \rightarrow \pi_{1}(\mathcal{T})$. Put $\Gamma=\pi\left(X_{0}\right) / \operatorname{Ker} \eta$ and $X=\operatorname{Ker} \eta \backslash \widetilde{X}_{0}$, where $\widetilde{X}_{0}$ is the universal covering graph of $X_{0}$. Then $X$ is a $\Gamma$-crystal lattice with $X_{0}$ as its quotient graph.

Lemma 4.5. There exists an affine isomorphism $\alpha: \Gamma \otimes \mathbb{R} / \Gamma \rightarrow \mathcal{T}$ such that $\alpha \circ \Phi^{\Gamma} \in C$.

Proof. Identify $\mathcal{T}$ with $H_{1}(\mathcal{T}, \mathbb{R}) / H_{1}(\mathcal{T}, \mathbb{Z})$ and let $\rho: \Gamma \rightarrow \pi(\mathcal{T})=H_{1}(\mathcal{T}, \mathbb{Z})$ be the isomorphism induced from $\eta$. Extend $\rho$ to the linear isomorphism $\rho_{\mathbb{R}}: \Gamma \otimes \mathbb{R} \rightarrow$ $H_{1}(\mathcal{T}, \mathbb{R})$ and get the affine isomorphism $\alpha: \Gamma \otimes \mathbb{R} / \Gamma \rightarrow H_{1}(\mathcal{T}, \mathbb{R}) / H_{1}(\mathcal{T}, \mathbb{Z})=\mathcal{T}$. Since $\rho_{\mathbb{R}} \circ \widetilde{\Phi}^{\Gamma}$ is a lift of $\alpha \circ \Phi^{\Gamma}$ and

$$
\rho_{\mathbb{R}}\left(\widetilde{\Phi}^{\Gamma}(\sigma x)\right)=\rho_{\mathbb{R}}\left(\widetilde{\Phi}^{\Gamma}(x)\right)+\rho(\sigma),
$$

we observe that $\alpha \circ \Phi^{\Gamma}$ is in $C$.

Put $\Phi_{0}=\alpha \circ \Phi^{\Gamma}$ and $g_{0}=c\left(\alpha^{*}\right)^{-1} g_{a l b}$, where $c$ is a positive constant with $v_{0}=\operatorname{vol}\left(c g_{\text {alb }}\right)$. Then, for a piecewise smooth map $\Phi \in C$ and a flat metric $g$ on $\mathcal{T}$ with $\operatorname{vol}(g)=v_{0}$, we have

$$
\begin{aligned}
E(\Phi, g) & =E\left(\alpha^{-1} \circ \Phi, \alpha^{*} g\right) \\
& \geq E\left(\Phi^{\Gamma}, \alpha^{*} g\right) \geq E\left(\Phi^{\Gamma}, c g_{a l b}\right)=E\left(\Phi_{0}, g_{0}\right) .
\end{aligned}
$$

The equality holds if and ony if $g=g_{0}$ and $\Phi$ is a translation of $\Phi_{0}$. This completes the proof of Theorem 2, which is restated as follows:

Theorem 4.6. Let $X_{0}$ be a finite connected weighted graph, $\mathcal{T}$ a torus, and $C$ a homotopy class of piecewise smooth maps from $X_{0}$ into $\mathcal{T}$ such that a map in $C$ induces a surjective homomorphism $\eta$ of $\pi_{1}\left(X_{0}\right)$ onto $\pi_{1}(\mathcal{T})$. For a given positive constant $v_{0}$, there exists a pair $\left(\Phi_{0}, g_{0}\right)$, consisting of a harmonic map $\Phi_{0} \in C$ and a flat metric $g_{0}$ on $\mathcal{T}$ such that $\operatorname{vol}\left(\mathcal{T}, g_{0}\right)=v_{0}$, satisfying

$$
E\left(\Phi_{0}, g_{0}\right) \leq E(\Phi, g)
$$

for every $\Phi \in C$ and every flat metric $g$ on $\mathcal{T}$ with $\operatorname{vol}(\mathcal{T}, g)=v_{0}$. The pair $\left(\Phi_{0}, g_{0}\right)$ coincides essentially with the pair consisting of the Albanese map and the Albanese metric associated with the $\Gamma$-crystal lattice $X=\operatorname{Ker} \eta \backslash \widetilde{X}_{0}$, where $\widetilde{X}_{0}$ is the universal covering graph of $X_{0}$.

Remark. It should be noted that the standard realization is not always a faithful expression of a given crystal lattice, although many examples indicate that it usually is, as will be seen in the next section. Actually if $\omega(e)=0$ for all $\omega \in H^{1}\left(X_{0}, \mathbb{R}\right)$, then the edge $e$ degenerates in the standard realization of the maximal abelian covering graph $X$ of $X_{0}$, and vice versa. We call such an edge degenerate. Observe that an edge $e$ is degenerate if and only if the graph obtained from $X_{0}$ by removing $e$ is not connected. This follows from the fact that there is a circuit in $X_{0}$ which contains an edge $e$ if and only if the graph obtained by removing $e$ is connected. The union of all the degenerate edges forms a forest, a disjoint union of trees, in $X$. By collapsing each component of the complement of the forest in $X_{0}$ to one point, we have a tree. 


\section{EXAMPLES}

Let us take a look at some of the classically known examples of crystal lattices. We shall see that the equilibrium configurations of those crystal lattices are just as described in their figures (Figure 2, 3, 4, 5). To explain this, we keep the notation in the previous section. We shall realize a crystal lattice $X$ in the subspace $\Pi=$ $(\operatorname{Ker} \rho)^{\perp}$, which is identified with $\Gamma \otimes \mathbb{R}$, of the Euclidean space $H_{1}\left(X_{0}, \mathbb{R}\right) \cong \mathbb{R}^{n}$. Throughout this section we assume $m_{V} \equiv 1$ and $m_{E} \equiv 1$.

We shall first explain a general algorithm to realize a generalized lattice $X$ in the Euclidean space $\Pi$. For this, take a maximal tree $T=\left(V_{T}, E_{T}\right)$ of $X_{0}$. Fix a base point $x_{0} \in T$. Then one can equip an orientation $E_{0}^{o}=\left\{e_{1}, \ldots, e_{n}, t_{1}, \ldots, t_{N-1}\right\}$ with

$$
\begin{aligned}
e_{1}, \ldots, e_{n} & \notin E_{T}, \\
t_{1}, \ldots, t_{N-1} & \in E_{T},
\end{aligned}
$$

such that, for every vertex $x$ in $X_{0}$, there exists a minimal path $c=\left(t_{i_{1}}, \ldots, t_{i_{a}}\right)$ in $T$ with $o(c)=x_{0}$ and $t(c)=x$. Let $c_{1}, \ldots, c_{n}$ be the basis of $H_{1}\left(X_{0}, \mathbb{R}\right)$ constructed in the proof of Lemma 4.2. Take a basis $\mathbf{c}_{1}, \ldots, \mathbf{c}_{n}$ of $\mathbb{R}^{n}$ such that $\left\langle\mathbf{c}_{i}, \mathbf{c}_{j}\right\rangle_{\mathbb{R}^{n}}=\Lambda_{i j}=$ $c_{i} \cdot c_{j}$. By identifying $\mathbf{c}_{i}$ with $c_{i}$, we have an isometry between $\mathbb{R}^{n}$ and $H_{1}\left(X_{0}, \mathbb{R}\right)$. The vectors $\mathbf{c}_{i}^{\prime}$ are the orthogonal projections of $\mathbf{c}_{i}$ to the $k$-dimensional subspace $\Pi(k=\operatorname{rank} \Gamma)$. Put

$$
\mathbf{b}_{i}=\sum_{l=1}^{n} u_{l}\left(t_{i}\right) \mathbf{c}_{l}^{\prime} \in \Pi \quad(i=1, \ldots, N-1) .
$$

Then we easily see that $\mathbf{b}_{i}$ corresponds to $d \widetilde{\Phi}^{\Gamma}\left(t_{i}\right) \in \Gamma \otimes \mathbb{R}$ through the isometry between $\Pi$ and $\Gamma \otimes \mathbb{R}$.

Let $\widetilde{T} \subset X$ be a lift of $T$, and let $\tilde{x}_{0} \in \widetilde{T}$ be the vertex with $\varphi\left(\tilde{x}_{0}\right)=x_{0}$. Taking the path $c$ joining $x_{0}$ and $x=\varphi(\tilde{x})$ as above, we find that

$$
\widetilde{\Phi}^{\Gamma}(\tilde{x})=\sum_{s=1}^{a} \mathbf{b}_{i_{s}} \quad\left(\widetilde{\Phi}^{\Gamma}\left(\tilde{x}_{0}\right)=\mathbf{o}\right) .
$$

In this way, we obtain the position vectors in $\Pi$ corresponding to the vertices in $\widetilde{T}$. We draw the line segments joining two points according to the incidence of vertices and edges in $\widetilde{T}$.

Put

$$
\mathbf{b}_{N-1+i}=\mathbf{c}_{i}^{\prime}-\sum_{l=1}^{N-1} f_{i, n+l} \mathbf{b}_{l} \quad(i=1, \ldots, n),
$$

where we should recall that

$$
c_{i}=e_{i}+\sum_{l=1}^{N-1} f_{i, n+l} t_{l}
$$

Therefore $\mathbf{b}_{N-1+i}$ gives the vector in $\Pi$ corresponding to $d \widetilde{\Phi}^{\Gamma}\left(e_{i}\right)$. Let $\tilde{e}_{i}$ be the lift of $e_{i}$ with $o\left(\tilde{e}_{i}\right) \in \widetilde{T}$. Then the figure consisting of the line segments joining $\widetilde{\Phi}^{\Gamma}\left(o\left(\tilde{e}_{i}\right)\right)$ and $\widetilde{\Phi}^{\Gamma}\left(o\left(\tilde{e}_{i}\right)\right)+\mathbf{b}_{N-1+i}$ together with the above figure yields a building block for the standard realizartion. Owing to the periodicity, the realization of $X$ is the union of its copies obtained by translations by $\mathbb{Z} \mathbf{c}_{1}^{\prime}+\cdots+\mathbb{Z} \mathbf{c}_{n}^{\prime}$. 
Example 5.1 (The standard lattice and the triangular lattice). The standard realization of the standard lattice is the hypercubic lattice (Figure 2), and that of the triangular lattice is the equilateral triangular lattice(Figure 3.a). Note that Figure 3.b gives a crystal lattice whose internal force is in equilibrium but where configuration is not.
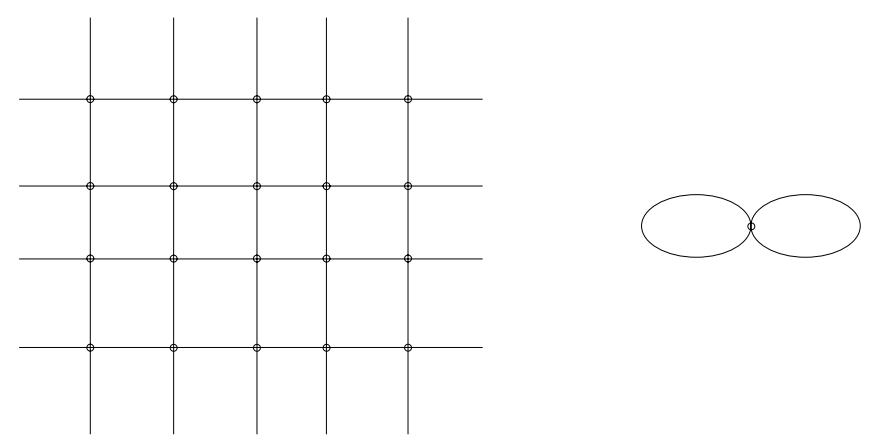

Figure 2. The standard lattice and its quotient graph
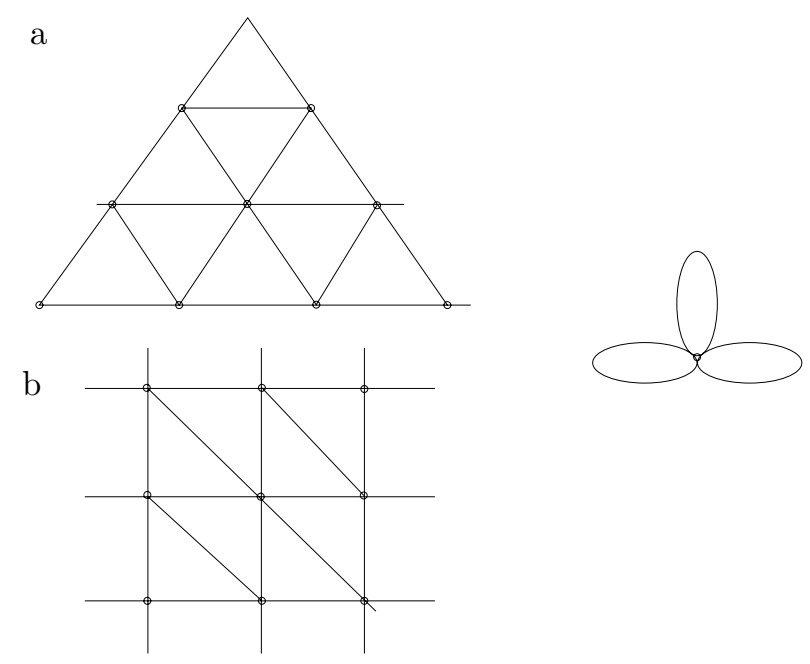

Figure 3. The triangular lattice (with equilibrium configuration and non equilibrium configuration)

Example 5.2 (The $n$-diamond lattice). Consider the maximal abelian covering graph $X_{0}^{a b}$ of the graph $X_{0}$ with two vertices joined by $n+1$ multiple edges $e_{1}, \ldots, e_{n+1}$ (Figure 1). Put $c_{i}=e_{i} \bar{e}_{n+1}$ for $i=1, \ldots, n$, which forms a $\mathbb{Z}$-basis of $H_{1}\left(X_{0}, \mathbb{Z}\right)$. We have

$$
F=\left(\begin{array}{rrrr|r}
1 & 0 & \cdots & 0 & -1 \\
0 & 1 & \cdots & 0 & -1 \\
\vdots & & \ddots & \vdots & \vdots \\
0 & 0 & \cdots & 1 & -1
\end{array}\right)
$$




$$
\Lambda=\left(\begin{array}{cccc}
2 & 1 & \cdots & 1 \\
1 & 2 & \cdots & 1 \\
\vdots & & \ddots & \vdots \\
1 & 1 & \cdots & 2
\end{array}\right), \quad U=\frac{1}{n+1}\left(\begin{array}{cccc}
n & -1 & \cdots & -1 \\
-1 & n & \cdots & -1 \\
\vdots & & \ddots & \vdots \\
-1 & -1 & \cdots & n
\end{array}\right)
$$

Therefore

$$
\begin{aligned}
\mathbf{b}_{i} & =\mathbf{c}_{i}-\frac{1}{n+1} \sum_{l=1}^{n} \mathbf{c}_{l} \text { for } i=1, \ldots, n, \\
\mathbf{b}_{n+1} & =\frac{-1}{n+1} \sum_{l=1}^{n} \mathbf{c}_{l} .
\end{aligned}
$$

Then we find that $\left\langle\mathbf{b}_{i}, \mathbf{b}_{j}\right\rangle=\delta_{i j}-\frac{1}{n+1}$. The realization of $X_{0}^{a b}$ is the figures consisting of $n+1$ vertices $\mathbf{b}_{i}$ of an equilateral $(n+1)$-simplex joined by line segments with the center of mass at the origin and its copies obtained by translation by $\sum_{i=1}^{n} \mathbb{Z} \mathbf{c}_{i}$. It is now easy to see that the $n$-diamond lattice graph is the hexagonal lattice graph when $n=2$ (Figure 4 ), and it gives the crystal structure of diamond when $n=3$.
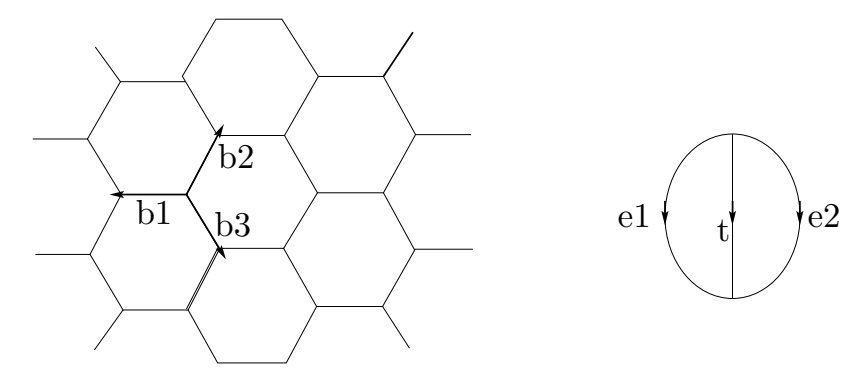

Figure 4. The hexagonal lattice and its quotient graph

Example 5.3 (The Kagome lattice). The Kagome lattice is the $\mathbb{Z}^{2}$-crystal lattice with the quotient graph $X_{0}$ which consists of three vertices joined by double edges (see Figure 5). Put $c_{1}=e_{1} \bar{t}_{1}, c_{2}=e_{2} \bar{t}_{2}, c_{3}=t_{1} e_{3} \bar{t}_{2}, c_{4}=t_{1} e_{4} \bar{t}_{2}$, which form a $\mathbb{Z}$-basis of $H_{1}\left(X_{0}, \mathbb{Z}\right)$. Here we realize first the maximal abelian covering graph $X_{0}^{a b}$ of $X_{0}$ and then the Kagome lattice as the image of the orthogonal projection of the realization of $X_{0}^{a b}$ to the 2-dimensional subspace $\Pi=\operatorname{Ker} \rho^{\perp}$.

We have

$$
\begin{gathered}
F=\left(\begin{array}{rrrr|rr}
1 & 0 & 0 & 0 & -1 & 0 \\
0 & 1 & 0 & 0 & 0 & -1 \\
0 & 0 & 1 & 0 & 1 & -1 \\
0 & 0 & 0 & 1 & 1 & -1
\end{array}\right), \\
\Lambda=\left(\begin{array}{rrrr}
2 & 0 & -1 & -1 \\
0 & 2 & 1 & 1 \\
-1 & 1 & 3 & 2 \\
-1 & 1 & 2 & 3
\end{array}\right), \quad U=\frac{1}{6}\left(\begin{array}{rrrr}
4 & -1 & 1 & -1 \\
-1 & 4 & -1 & -1 \\
1 & -1 & 4 & -2 \\
1 & -1 & -2 & 4
\end{array}\right) .
\end{gathered}
$$



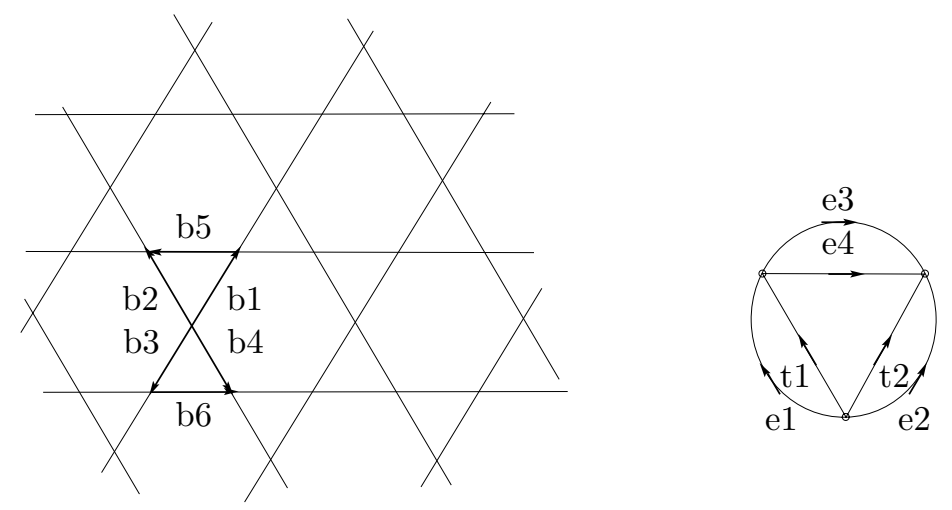

Figure 5. The Kagome lattice and its quotient graph

1) The maximal abelian covering graph $X_{0}^{a b}(n=k=4)$.

Let $\mathbf{c}_{i} \in \mathbb{R}^{4}$ be the vector corresponding to $c_{i}$ for $i=1,2,3,4$, so that $\left(\left\langle\mathbf{c}_{i}, \mathbf{c}_{j}\right\rangle_{\mathbb{R}^{4}}\right)=\Lambda$. By simple computation, we have

$$
\begin{aligned}
& \mathbf{b}_{1}=\frac{1}{6}\left(-2 \mathbf{c}_{1}-\mathbf{c}_{2}+\mathbf{c}_{3}+\mathbf{c}_{4}\right), \\
& \mathbf{b}_{2}=\frac{1}{6}\left(-\mathbf{c}_{1}-2 \mathbf{c}_{2}-\mathbf{c}_{3}-\mathbf{c}_{4}\right), \\
& \mathbf{b}_{3}=\frac{1}{6}\left(4 \mathbf{c}_{1}-\mathbf{c}_{2}+\mathbf{c}_{3}+\mathbf{c}_{4}\right), \\
& \mathbf{b}_{4}=\frac{1}{6}\left(-\mathbf{c}_{1}+4 \mathbf{c}_{2}-\mathbf{c}_{3}-\mathbf{c}_{4}\right) .
\end{aligned}
$$

The building block of the realization of $X_{0}^{a b}$ consists of the two triangles $\triangle \mathbf{o b}_{1} \mathbf{b}_{2}$ and $\triangle \mathbf{o b} \mathbf{b}_{3} \mathbf{b}_{4}$, and the realization of $X_{0}^{a b}$ is the union of their copies obtained by translatation by $\sum \mathbb{Z} \mathbf{c}_{i}$.

2) The Kagome lattice $(n=4, k=2)$.

A basis of $\Gamma$ is given by $\rho\left(c_{1}\right)=\sigma_{1}$ and $\rho\left(c_{2}\right)=\sigma_{2}$. Since $\rho\left(c_{4}\right)=0$ and $\rho\left(c_{3}\right)=\sigma_{2}-\sigma_{1}$, Ker $\rho=\operatorname{Span}\left\{c_{4}, c_{1}-c_{2}+c_{3}\right\}$. The Kagome lattice is realized in the subspace $\Pi=\operatorname{Ker} \rho^{\perp} \subset \mathbb{R}^{4}$, and the action of $\Gamma$ is generated by translation of the orthogonal projection $\mathbf{c}_{i}^{\prime}$ of $\mathbf{c}_{i}$ for $i=1,2$ to the subspace $\Pi$. The orthogonal projection of $\mathbf{b}_{i}$ in (1) to $\Pi$ gives the realization of the vertices of the building block of the Kagome lattice in $\Pi$, which we also denote by $\mathbf{b}_{i}$. They are given by

$$
\begin{aligned}
& \mathbf{b}_{1}=v_{1}\left(t_{1}\right) \mathbf{c}_{1}^{\prime}+v_{2}\left(t_{1}\right) \mathbf{c}_{2}^{\prime}=-\frac{1}{2} \mathbf{c}_{1}^{\prime}, \\
& \mathbf{b}_{2}=v_{1}\left(t_{2}\right) \mathbf{c}_{1}^{\prime}+v_{2}\left(t_{2}\right) \mathbf{c}_{2}^{\prime}=-\frac{1}{2} \mathbf{c}_{2}^{\prime}, \\
& \mathbf{b}_{3}=\mathbf{c}_{1}^{\prime}+\widetilde{\Phi}\left(p_{1}\right)-\widetilde{\Phi} i(o)=\frac{1}{2} \mathbf{c}_{1}^{\prime}, \\
& \mathbf{b}_{4}=\mathbf{c}_{2}^{\prime}+\widetilde{\Phi}\left(p_{2}\right)-\widetilde{\Phi}(o)=\frac{1}{2} \mathbf{c}_{2}^{\prime} .
\end{aligned}
$$


By using the relations

\begin{tabular}{c|rrrc}
\hline$\langle\rangle$, & $c_{4}$ & $c_{1}$ & $c_{2}$ & $c_{1}-c_{2}+c_{3}$ \\
\hline$c_{4}$ & 3 & -1 & 1 & 0 \\
$c_{1}$ & -1 & 2 & 0 & 1 \\
$c_{2}$ & 1 & 0 & 2 & -1 \\
$c_{1}-c_{2}+c_{3}$ & 0 & 1 & -1 & 3 \\
\hline
\end{tabular}

we have

$$
\begin{aligned}
\mathbf{c}_{1}^{\prime} & =\mathbf{c}_{1}-\frac{1}{3}\left\langle\mathbf{c}_{1}, \mathbf{c}_{1}-\mathbf{c}_{2}+\mathbf{c}_{3}\right\rangle\left(\mathbf{c}_{1}-\mathbf{c}_{2}+\mathbf{c}_{3}\right)-\frac{1}{3}\left\langle\mathbf{c}_{1}, \mathbf{c}_{4}\right\rangle \mathbf{c}_{4} \\
& =\frac{1}{3}\left(2 \mathbf{c}_{1}+\mathbf{c}_{2}-\mathbf{c}_{3}+\mathbf{c}_{4}\right), \\
\mathbf{c}_{2}^{\prime} & =\mathbf{c}_{2}-\frac{1}{3}\left\langle\mathbf{c}_{2}, \mathbf{c}_{1}-\mathbf{c}_{2}+\mathbf{c}_{3}\right\rangle\left(\mathbf{c}_{1}-\mathbf{c}_{2}+\mathbf{c}_{3}\right)-\frac{1}{3}\left\langle\mathbf{c}_{2}, \mathbf{c}_{4}\right\rangle \mathbf{c}_{4} \\
& =\frac{1}{3}\left(\mathbf{c}_{1}+2 \mathbf{c}_{2}+\mathbf{c}_{3}-\mathbf{c}_{4}\right) .
\end{aligned}
$$

Notice that $\triangle \mathbf{o} \mathbf{c}_{1}^{\prime} \mathbf{c}_{2}^{\prime}$ forms an equilateral triangle with edges of length $2 / \sqrt{3}$. The building block of the realization of the Kagome lattice consists of the two triangles $\frac{1}{2} \triangle \mathbf{o c}_{1}^{\prime} \mathbf{c}_{2}^{\prime}$ and $\frac{-1}{2} \triangle \mathbf{o c}_{1}^{\prime} \mathbf{c}_{2}^{\prime}$, and the realization of the Kagome lattice is the union of their copies obtained by translation by $\mathbb{Z} \mathbf{c}_{1}^{\prime}+\mathbb{Z} \mathbf{c}_{2}^{\prime}$ in the subspace $\Pi$.

\section{REFERENCES}

1. B. Bollobas, Graph Theory, Springer GTM 63. MR 80j:05053

2. J. Eells, Jr. and J.H. Sampson, Harmonic mappings of Riemannian manifolds, Amer. J. Math. 86(1964) 109-160. MR 29:1603

3. N. Ejiri, The second variation formula of the energy function on the Teichmüller space and the Morse index of compact minimal surfaces in tori, preprint.

4. M. Gromov and R. Schoen, Harmonic maps into singular spaces and p-adic superrigidity for lattices in groups of rank one, Publ. Math. IHES 76(1992) 165-246. MR 94e:58032

5. J. Jost, Equilibrium maps between metric spaces, Calc. Var. 2(1994) 173-204. MR 98a:58049

6. J. Jost, Convex functionals and generalized harmonic maps into spaces of non positive curvature, Comm. Math. Helv. 70(1995) 659-673. MR 96j:58043

7. M. Kotani and T. Sunada, Jacobian tori associated with a finite graph and its abelian covering graphs, preprint.

8. M. Kotani and T. Sunada, Albanese maps and off diagonal long time asymptotics for the heat kernel, preprint.

9. T. Nagano and B. Smyth, Minimal varieties and harmonic maps in tori, Comm. Math. Helv. 50(1975), 249-265. MR 52:11797.

10. H. Urakawa, A discrete analogue of the harmonic morphism and Green kernel comparison theorems, preprint.

11. E.A. Wood, Crystals and Light, An Intorduction to Optical Crystallography, Dover, 1977.

12. J. Eells and B. Fuglede, Harmonic maps between Riemannian polyhedra, a monograph to be published.

Mathematical Institute, Graduate School of Science, Tôhoku University, Aoba, SENDAI 980-8578, JAPAN

E-mail address: kotani@math.tohoku.ac.jp

Mathematical Institute, Graduate School of Sciences, Tôhoku University, Aoba, SENDAI 980-8578, JAPAN

E-mail address: sunada@math.tohoku.ac.jp 